\title{
Variations in the ultrastructure of human nasal cilia including abnormalities found in retinitis pigmentosa
}

\author{
BERNARD FOX ${ }^{1}$, TERENCE B BULL 1 , AND GEOFFREY B ARDEN 2 \\ From the ${ }^{1}$ Department of Histopathology, Charing Cross Hospital Medical School, Fulham Palace Road, \\ London W6 8RF, and ${ }^{2}$ Department of Visual Science, Institute of Ophthalmology, Judd Street, London \\ WC1, UK
}

SUMmARY The electron microscopic structure of cilia from the inferior turbinate of the nose was studied in 12 adults, four with chronic sinusitis, one with allergic rhinitis, two with bronchiectasis, three with deviated nasal septum, and two normals. The changes are compared with those found in nasal cilia in 14 patients with retinitis pigmentosa.

There were compound cilia in the seven cases with chronic sinusitis, allergic rhinitis, and bronchiectasis but, apart from this, the structure of the cilia was similar in all 12 cases. There were variations in the microtubular pattern in about $4 \%$ of cilia, dynein arms were not seen in $4 \%$, and in the rest an average of 5-6 dynein arms were seen in each cilium. The orientation of the cilia was 0 to $90^{\circ}$.

In the retinitis pigmentosa patients there was a highly significant increase in cilial abnormalities.

The establishment on a quantitative basis of the variations in normal structure of nasal cilia facilitated the recognition of an association between cilial abnormalities and retinitis pigmentosa and should help in the identification of associations that may exist between cilial abnormalities and other diseases.

In a recent study of ultrastructural abnormalities in nasal cilia in retinitis pigmentosa ${ }^{1}$ we found it essential to establish in some quantitative form the type and range of abnormalities present in nasal cilia from a group of control subjects. This information will also be useful in the investigation of chronic lung diseases ${ }^{2-6}$ and sterility in men, ${ }^{7} 8$ in which there are known abnormalities in cilia, and should enable one to recognise associations of cilial abnormalities with other diseases.

The electron microscopic structure of normal cilia and various abnormalities found are described, and quantitative measurements of the various changes are made and compared with those found in patients with retinitis pigmentosa.

\section{Subjects studied and methods}

Twelve subjects ( 8 men, 4 women) were studied: two healthy volunteers, three patients with deviated

Received for publication 25 September 1979 nasal septum but with no other nasal abnormalities, four with chronic sinusitis, one with allergic rhinitis, and two with bronchiectasis. Their ages ranged from 21 to 66 years.

In a similar study in patients with retinitis pigmentosa, we examined 14 patients ( 9 men, 5 women) aged 22 to 59 years, who had been investigated at the retinal, genetic, and electrodiagnostic clinics of Moorfields Eye Hospital. Six of the patients had been called 'Usher's syndrome' (autosomal recessive retinitis pigmentosa with deafness ${ }^{9}$ ), although in some cases the deafness was not profound or was of very early onset. In two cases the inheritance was autosomal dominant, and in three X-linked, all without deafness, although audiometry has not been performed. In three cases the retinitis pigmentosa seemed to be sporadic and associated with deafness. In one of these, deafness was inherited dominantly but the propositus was the only member of the family with retinitis pigmentosa. On this basis and considering the nature of the disease in each patient, we classified these cases as one dominant and two as recessive 
forms of inheritance. Thus there were eight cases of autosomal recessive, three cases of autosomal dominant, and three with X-linked inheritance.

Samples were taken from the inferior surface of the inferior nasal turbinate as far back as possible in the nose. In the two healthy volunteers and in one patient with bronchiectasis and 14 patients with retinitis pigmentosa, they were taken under local anaesthesia by spraying $4 \%$ lignocaine hydrochloride directly on to the surface of the turbinate and using Tilley-Henckel forceps to obtain the biopsy specimen. In the rest, the biopsies were performed during the course of operations on the nose performed under general anaesthesia.

The tissue was immediately pinned flat on a piece of cork with the ciliated surface uppermost and placed in Karnovsky fixative. After 1 hour the excess connective tissue was trimmed away and the tissue was cut into small pieces and allowed to fix overnight. In the latter part of the study $2 \mathrm{mmol} \mathrm{mag}$ nesium sulphate was added to the fixative to improve the visibility of the dynein arms. After washing in cacodylate sucrose buffer at $4^{\circ} \mathrm{C}$ the specimen was post-fixed in $1 \%$ aqueous osmium tetroxide for 1 hour at $4^{\circ} \mathrm{C}$ followed by washing in distilled water for 5 minutes and block staining in $2 \%$ aqueous uranyl acetate for about 1 hour. The tissue was dehydrated to absolute alcohol and placed in two changes of epoxy propane for 15 minutes each and then in warmed $\left(60^{\circ} \mathrm{C}\right)$ Araldite for 1 hour. The tissue was polymerised in fresh Araldite at $60^{\circ} \mathrm{C}$ for at least 48 hours. Sections were cut with gold-blue interference colour for low power and silver-gold for study of internal structure on an Ultramicrotome LKB3. Sections were contrasted with $2 \%$ uranyl acetate and lead citrate and examined in a Philips 201 electron microscope fitted with a goniometer stage. To assess the internal axonemal structure electron micrographs of transverse sections of cilia were taken with a final magnification of 65-180 000. Only those cilia in which the microtubular pattern was clearly seen were counted. In cases in which there were few precisely transverse sections of cilia the gonioscopic stage was used to ensure that a clear outline was obtained. The microtubular structure and the number of dynein arms were counted, and the diameter of the axoneme was measured. To assess the orientation of one cilium to another, a line was drawn which passed through the centre of each central singlet, and the angle that each line made to one arbitrarily chosen line on the same photomicrograph was measured. To determine the possible sampling error in the search for compound cilia, electron micrographs were taken at a final magnification of 10800 so that longitudinal sections through the cells and transverse sections through the cilia were obtained (Fig. 1). The total length of the ciliated surface in the section and the length of a single ciliated cell

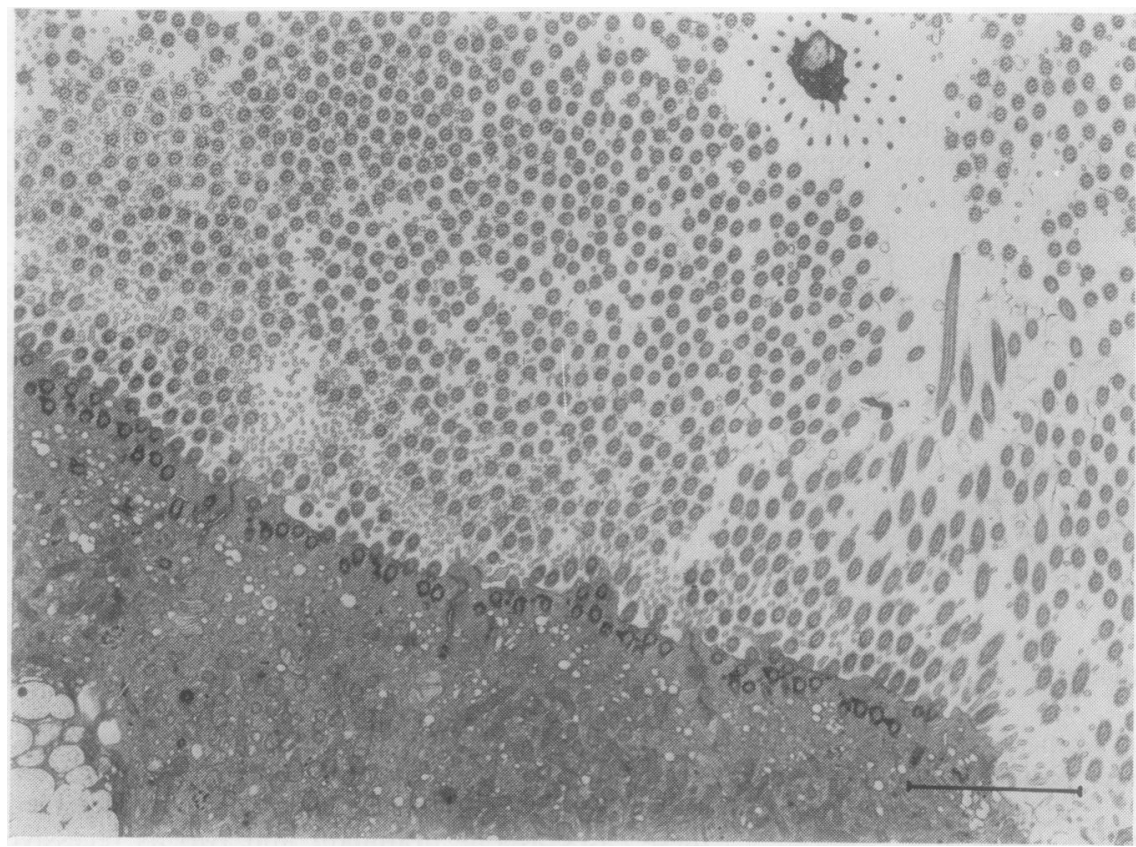

Fig. 1 Longitudinal section of nasal mucosa with transverse sections of cilia. Bar mark $3 \mu \mathrm{m}$. 
Table 1

\begin{tabular}{|c|c|}
\hline $\begin{array}{l}\text { Percentage abnormal microtubular arrangements } \\
\mathrm{N}=12 \quad \mathrm{X}=4.2 \% \\
\text { range } 0-20 \%\end{array}$ & $\mathrm{SE}=1.6 \%$ \\
\hline $\begin{array}{l}\text { Percentage dynein arms } \\
\begin{array}{l}* \mathrm{~N}=10 \\
\text { range } 18 \cdot 3-51 \cdot 6 \%\end{array}\end{array}$ & $\mathrm{SE}=3.2 \%$ \\
\hline $\begin{array}{l}\text { Diameter of shaft of axoneme } \\
N=12 \quad \begin{array}{l}\bar{X}=221 \mathrm{~nm} \\
\text { range } 196-259 \mathrm{~nm}\end{array}\end{array}$ & $\mathrm{SE}=5.8 \mathrm{~nm}$ \\
\hline
\end{tabular}

*The two cases of bronchiectasis were excluded because of the reported association of loss of dynein arms with bronchiectasis.

\begin{tabular}{lcc}
\hline Percentage abnormal microtubular arrangements & in retinitis pigmentosa \\
$\mathrm{N}=14$ & $\mathrm{X}=25.5 \%$ & $\mathrm{SE}=3.09 \%$ \\
& range $6-44 \%$ & \\
$\mathrm{~N}=3$ & (subgroup with dominant inheritance) \\
& $\mathrm{X}=23.73 \%$ & $\mathrm{SE} 4.8 \%$ \\
$\mathrm{~N}=3$ & (subgroup with X-linked inheritance) \\
\hline
\end{tabular}

associated with the largest number of transverse sections of cilia were measured. The number of cilia cut in true transverse section associated with this cell were counted. Thus we estimated the total number of cilia in the sample; this method overestimates the number of cilia. The number of compound cilia within the measured length of cells were counted.

\section{Results}

STRUCTURE OF NORMAL CILIA (see Table 1)

The internal structure of the axoneme of cilia has the classical $9+2$ microtubular pattern (Fig. 2) described by Fawcett and Porter. ${ }^{10}$ The central microtubules are singlets, and the peripheral nine microtubules are doublets with a figure-of-eight appearance; there is one complete microtubule called subfibre A, and the other (subfibre B) shares a common wall with $\mathrm{A}$. There are two short, diverging arms projecting from subfibre A (dynein arms). When sections are viewed from the base of the cilium to the tip the arms are in a clockwise direction (Fig. 2). The number of dynein arms are presented in Table 1. Microtubule $A$ is connected by radial spokes (Fig. 2) to a sheath that surrounds the central two singlets; it is often difficult clearly to identify the radial spokes and central sheaths. However, there were no cilia in which the radial spokes appeared to be entirely absent. Proximally, the central singlet microtubules end a short distance before the axoneme meets the surface of the cell (Fig. 3). If transverse sections are taken from below the end of the singlets and above the surface of the cell no central singlets will be seen, that is, a $9+0$ pattern (Fig. 2).

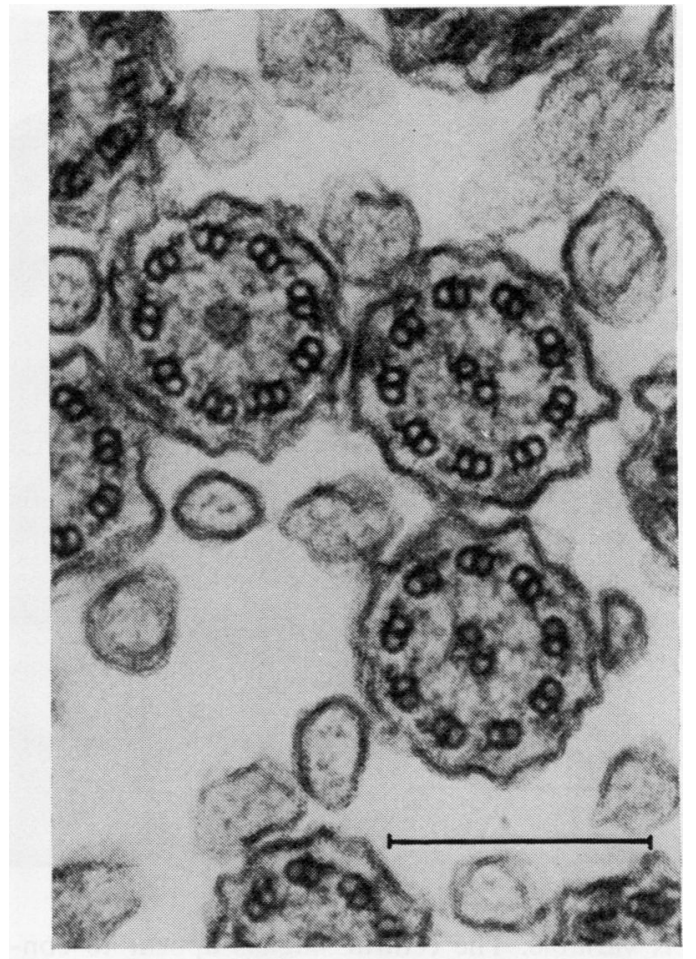

(a)

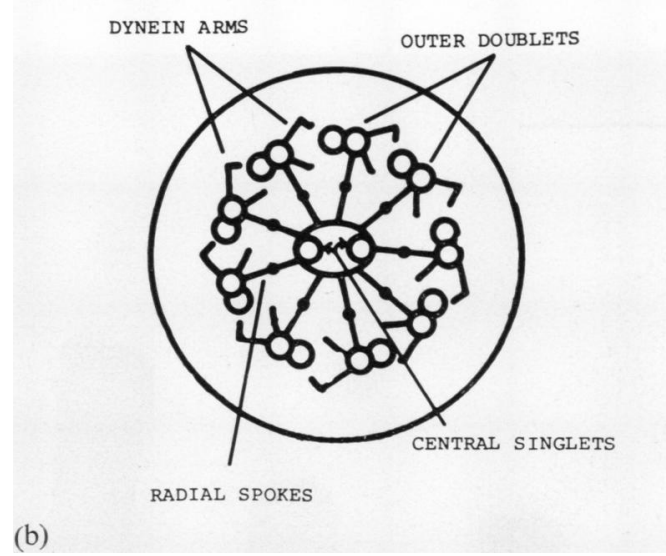

Fig. 2 (a) Transverse sections of nasal cilia showing normal $9+2$ and $9+0$ microtubular arrangements with dynein arms and radial spokes. (b) Diagram illustrating internal structure of cilia. Bar mark 0.25 $\mu \mathrm{m}$.

The transverse diameter of the shaft of the axoneme is about $220 \mathrm{~nm}$ (see Table 1). The axoneme starts to narrow at about $0.5 \mu \mathrm{m}$ from the tip (Fig. 4). The microtubular pattern at and around the 


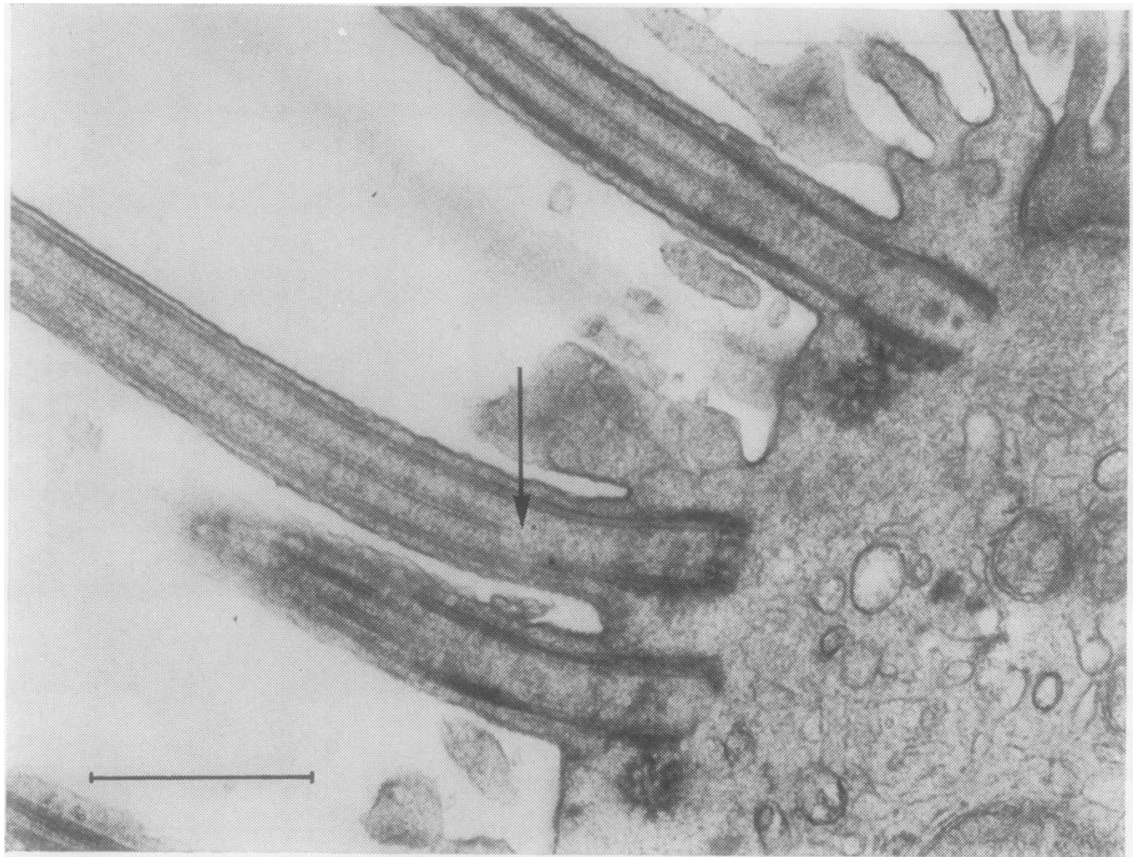

Fig. 3 Longitudinal section of nasal cilia showing end of central singlets above cell surface (arrow) and basal bodies. Bar. mark $0.5 \mu \mathrm{m}$.

tip is variable. The central singlets appear to continue to the tip, but the subfibre $B$ ends close to the tip and the subfibre A continues to the tip but loses its dynein arms so that in transverse section there are nine peripheral A microtubules with two central singlets (Fig. 5). In some cilia there appears to be

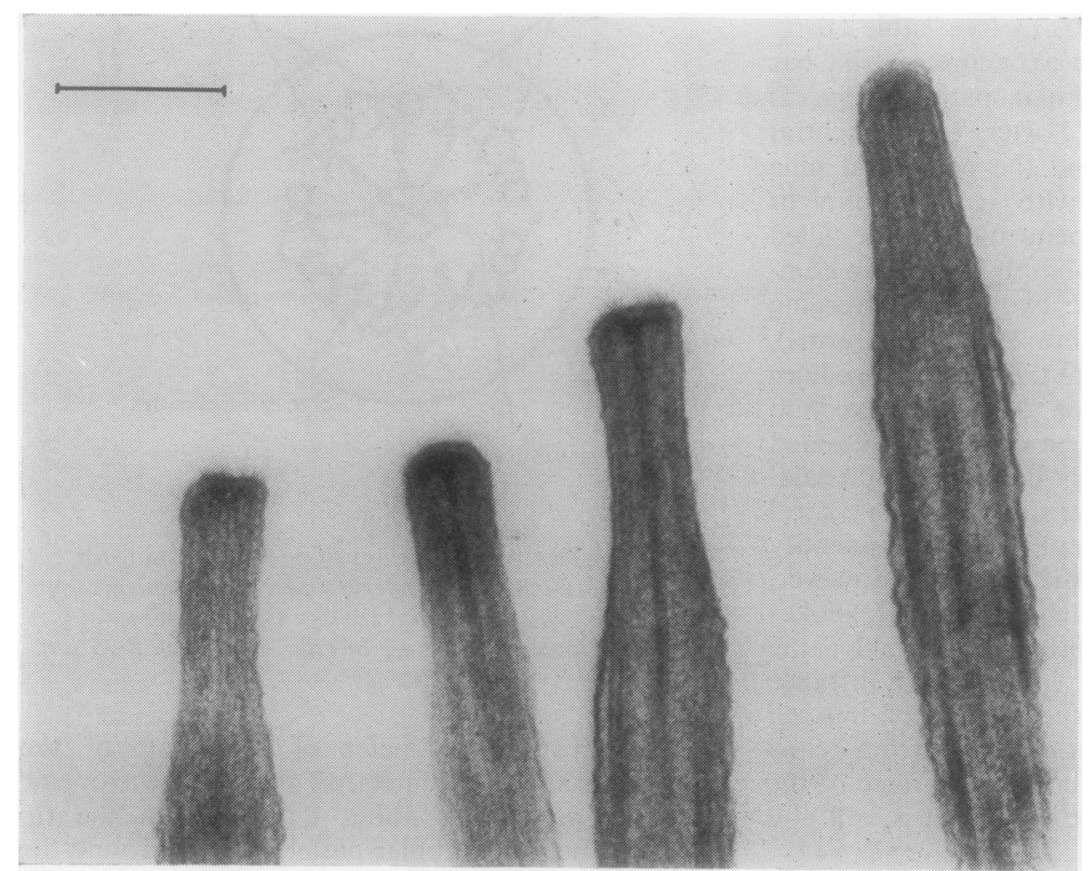

Fig. 4 Longitudinal sections of tips of nasal cilia. Bar mark 0.25 $\mu \mathrm{m}$. 


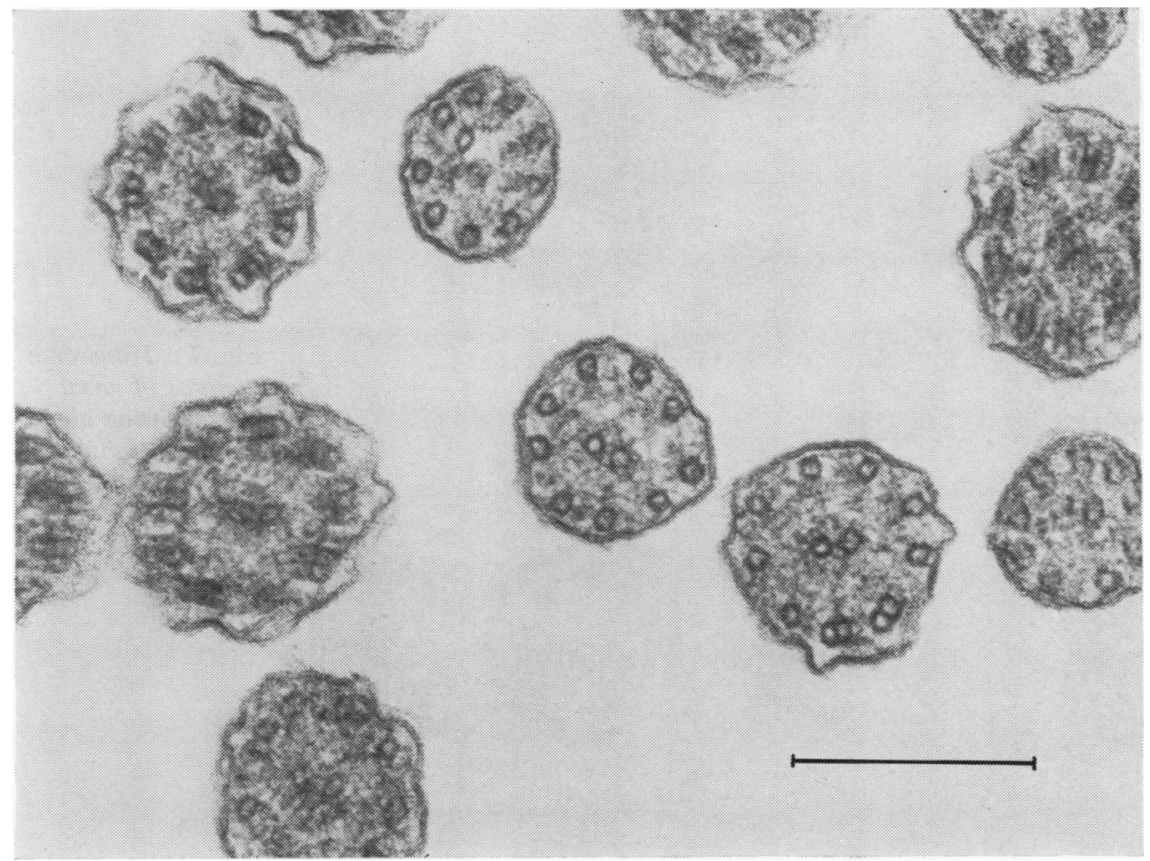

Fig. 5 Transverse sections from tip of nasal cilia showing microtubular arrangements with nine outer and two central singlets, and seven outer singlets, two doublets, and two central singlets. Bar mark $0 \cdot 25 \mu \mathrm{m}$.

a symmetrical loss of B fibres, so that some of the outer doublets are replaced by singlets (Fig. 5).

The orientation of the cilia varies from 0 to $90^{\circ}$ (Fig. 6). There was no difference in the distribution of orientation in the patients with compound cilia and the rest.

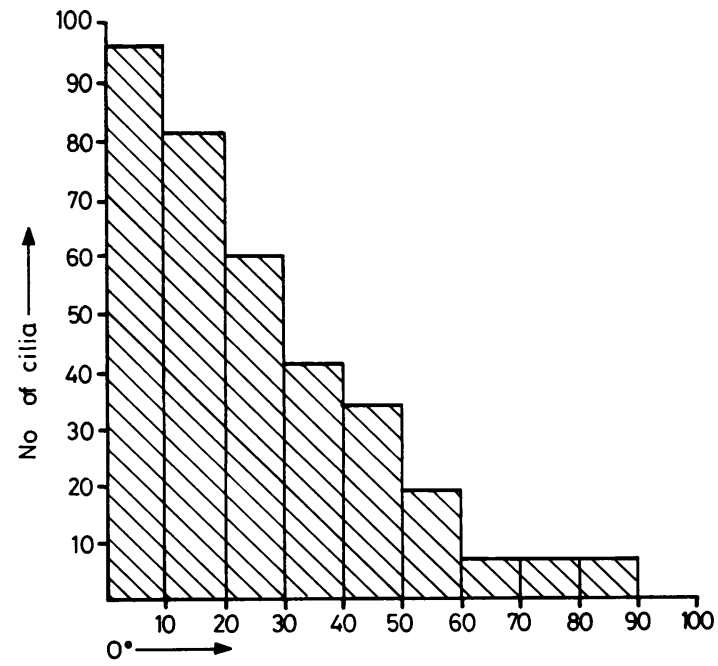

Fig. 6 Histogram showing range of orientation of nasal cilia from 12 cases.
VARIATIONS FROM NORMAL

Microtubular structures (Table 1 and Fig. 7)

There were 13 cilia with abnormal microtubular structures out of 311 transverse sections of cilia counted. The microtubular abnormalities found were 10 outer doublets, accessory singlet microtubules in peripheral ring, loss of one peripheral doublet, and irregularly arranged outer doublets.

\section{Compound cilia}

Compound cilia are cilia that have more than one set of $9+2$ microtubules within the axoneme (Fig. 8). There was considerable variation in the arrangement and number of microtubules from 2 to 20 structures, but in some the microtubular pattern was incomplete. In Table 2 the statistics on the incidence of compound cilia are presented. For each patient we were able to determine the proportion of compound cilia and thus obtain the standard error of the mean (SEM) which should be normally distributed. We were then able to make predictions about the number of compound cilia we expect to see in the normal group, on the assumption that the differences between the two groups are due solely to sampling. The number of compound cilia that should have been present in the normals (that is, those cases with no nasal abnormalities) if this was a sampling error were 11 using a simple average, and 17 using the 

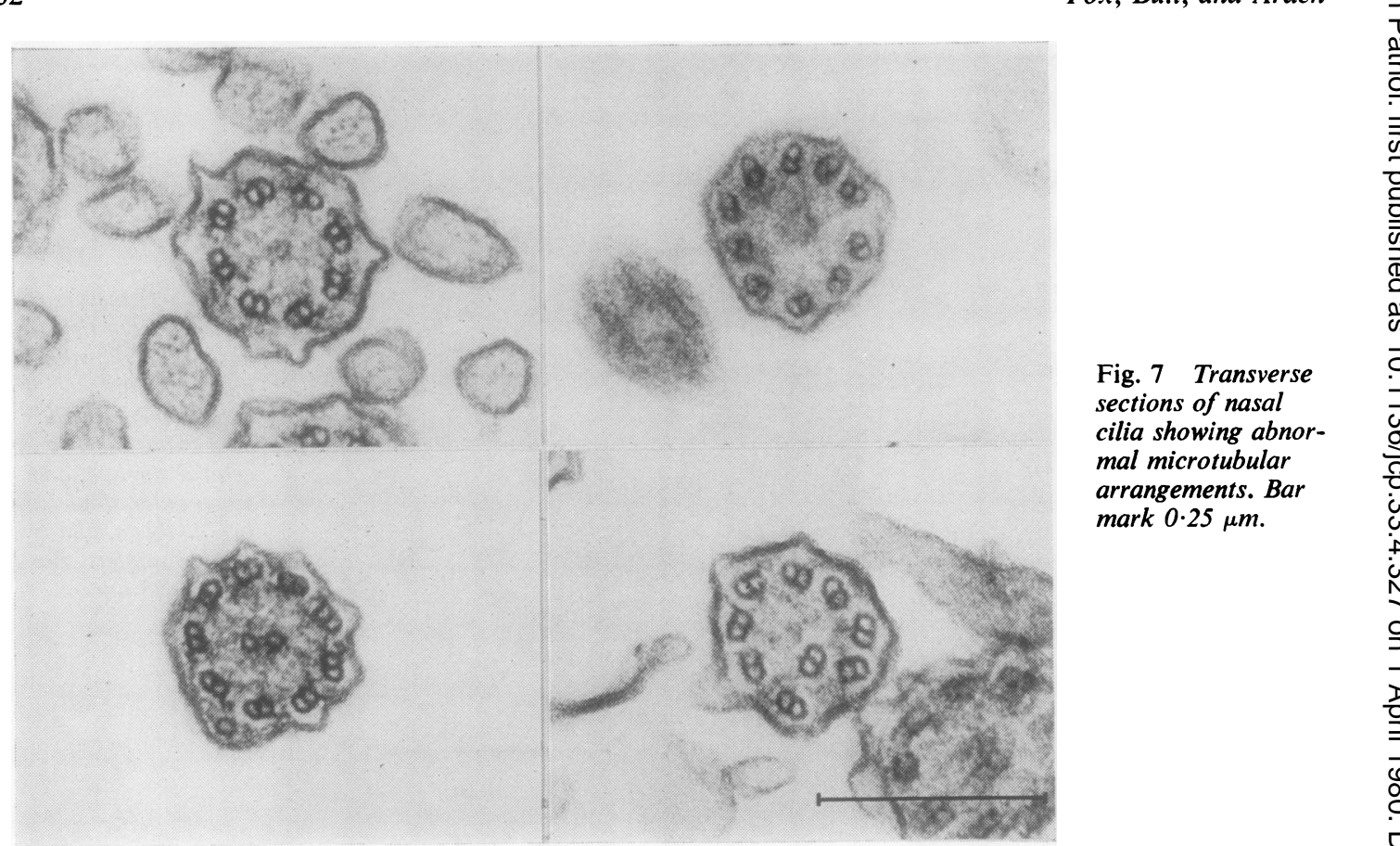

Fig. 7 Transverse sections of nasal cilia showing abnormal microtubular arrangements. Bar mark $0 \cdot 25 \mu \mathrm{m}$.
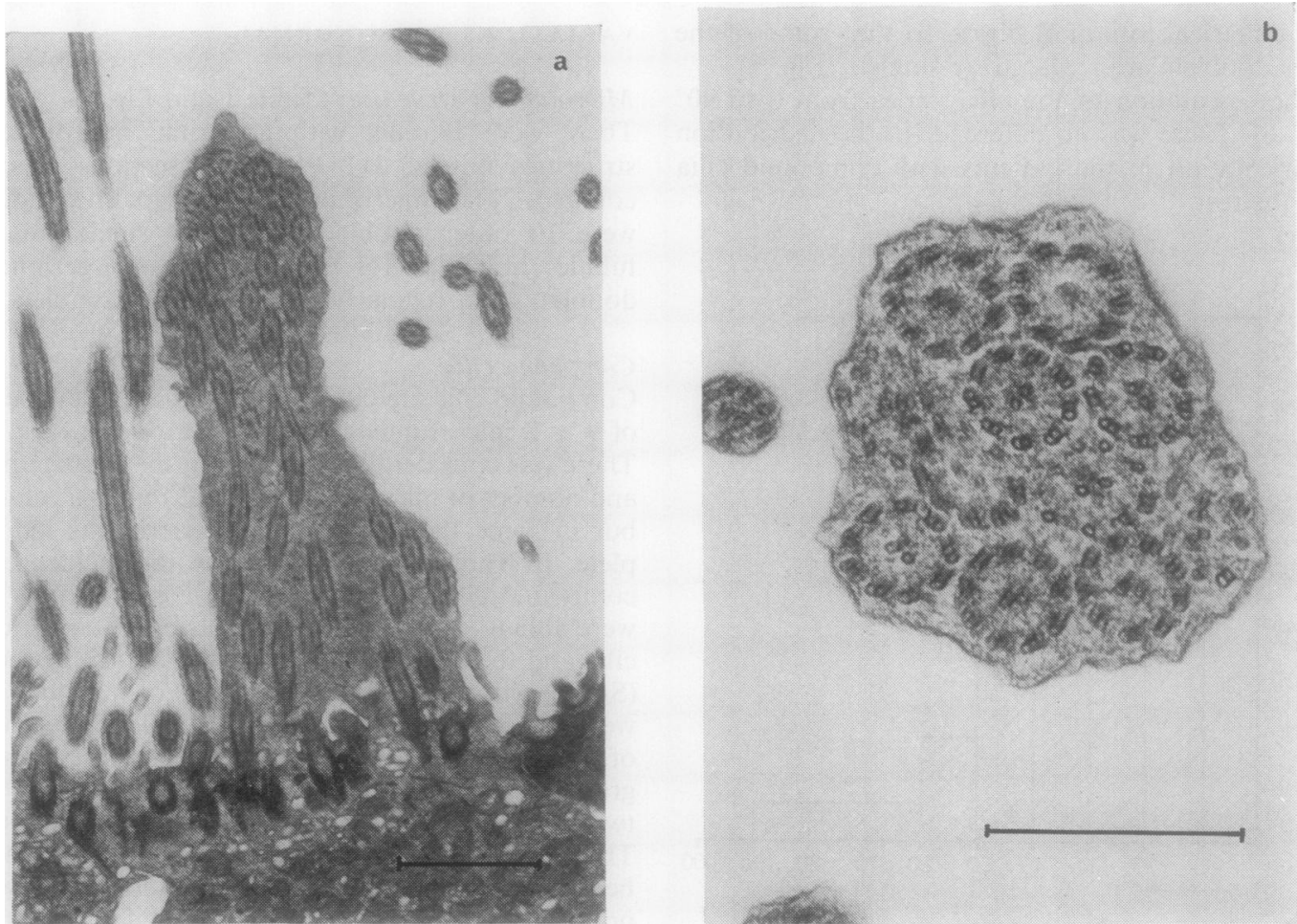

Fig. 8 Longitudinal and transverse sections of compound nasal cilia. Bar mark (a) $1 \mu \mathrm{m}$ and (b) $0 \cdot 25 \mu \mathrm{m}$. 
Table 2 Compound cilia

\begin{tabular}{|c|c|c|c|c|}
\hline & & Normal & Abnormal & Retinitis pigmentosa \\
\hline $\begin{array}{l}\text { Number } \\
\text { Total length of surface examined }(\mu \mathrm{m}) \\
\text { Length of ciliated cell }(\mu \mathrm{m}) \\
\text { Cilia/cell } \\
\text { Estimated number of cilia in sample } \\
\text { Number of compound cilia in sample } \\
\text { Percentage of compound cilia } \\
\text { Expected number of compound cilia in normal }\end{array}$ & $\begin{array}{l}\text { Mean } \\
\text { Range } \\
\text { Mean } \\
\text { Range } \\
\overline{\mathbf{X}} \\
\text { SEM }\end{array}$ & $\begin{array}{l}5 \\
597 \\
7 \cdot 5 \\
4 \cdot 0-9 \cdot 9 \\
62 \\
36-125 \\
5574 \\
0 \\
0 \\
\overline{11} \text { (from } \\
17 \text { (from } \\
2-32 \text { (士 }\end{array}$ & $\begin{array}{l}7 \\
935 \\
6 \cdot 6 \\
4 \cdot 4-7 \cdot 7 \\
84 \\
34-130 \\
10480 \\
20 \\
0 \cdot 31 \\
0 \cdot 14 \\
\text { verage) } \\
(\bar{X})\end{array}$ & $\begin{array}{l}7 \\
734 \\
7 \cdot 3 \\
5 \cdot 0-10 \cdot 1 \\
89 \\
23-113 \\
12068 \\
39 \\
0 \cdot 86 \\
0 \cdot 32 \\
21 \text { (from simple average) } \\
55 \text { (from value of } \bar{X}) \\
13-96( \pm 2 \text { SEM) }\end{array}$ \\
\hline
\end{tabular}

value of the mean (2-32 with 2 SEM). Thus we are able to reject this hypothesis.

\section{ABNORMALITIES IN RETINITIS PIGMENTOSA}

\section{Microtubular structures (Table 1)}

In the 14 patients with retinitis pigmentosa, 104 out of 348 cilia showed abnormal microtubular structures. The abnormalities affected the outer doublets, which varied in number from 2 to 10 and frequently were irregularly arranged. There were accessory singlet microtubules in the outer ring and variations in the central singlets. Sometimes there was only one central singlet, and in two cilia there were four and in one cilium five central singlets.

\section{Compound cilia (Table 2)}

There was a higher incidence of compound cilia in the patients with retinitis pigmentosa with no evidence of nasal abnormalities than in the control patients with nasal abnormalities. The number of compound cilia that should have been present in the normals if this was a sampling error was even more striking than in those with nasal abnormalities.

\section{Discussion}

As far as we can ascertain there have been no previous attempts to establish in any quantitative way the variations that may be found in the ultrastructure of normal human cilia. We have examined nasal cilia because they are easily obtainable under local anaesthesia and, apart from slight discomfort and occasionally a small nose bleed, it is a harmless procedure. It is important that the biopsy should be taken as far back in the nose as possible. We have found that up to one-half of the front part of the inferior nasal turbinate is lined by stratified squamous epithelium. In conditions in which there are generalised abnormalities of cilia, changes in the cilia from the nose are identical with those seen in bronchi. ${ }^{2} 6$

Most cilia have the normal $9+2$ microtubular structure but an occasional cilium may be abnormal. Although the average is about $4 \%$, in one healthy individual $20 \%$ of cilia were abnormal.

It is important to realise that in transverse sections from near the tip and base of the axoneme the normal $9+2$ structure is not present (Figs 2 and 5) and these should not be confused with a pathological abnormality of microtubular structure. The absence of the central singlets, that is, the $9+0$ pattern, has been regarded as a pathological change, ${ }^{411} 12$ but one could accept this only if it was shown that the central singlets were absent from the shaft of the axoneme and not just in sections taken from the basal region.

Our findings (Table 2) clearly show that compound cilia must be regarded as a pathological change. How compound cilia are formed is not clear, but it seems likely that they may arise by fusion of axonemes or by more than one $9+2$ microtubular structure extending into a single cytoplasmic protrusion. However, more complex mechanisms may be involved because although there may be up to 20 microtubular structures in a compound cilium we have not seen more than three basal bodies associated with one compound cilium; nor have we seen more than two axonemes fusing together. It is possible that compound cilia are the result of abnormal regeneration after local damage of cilia as they are frequently seen in association with infections ${ }^{313}$ and in chronic bronchitis and carcinoma of bronchus. 111214 However, they may also indicate more generalised damage to cilia as they were present in the nose in two patients with bronchiectasis in this series, and in patients with retinitis pigmentosa. ${ }^{1}$

In the group of retinitis pigmentosa patients, nine of whom suffered from deafness, there was a highly 
significant increase in cilial abnormalities when compared with the control group. As the outer limbs of photoreceptors are modified cilia ${ }^{15}$ and the sensory epithelium of the inner ear is derived from ciliated epithelium, it seems possible that the cilial abnormalities are related to the disease process in the eye and ear. It could be that there is a generalised defect in cilia, and that the loss of photoreceptors that occurs in retinitis pigmentosa is due to the same basic cause. Our results do not show immediately how the abnormality detected could produce retinitis pigmentosa. The growth of rod discs or the provision of metabolities for the rod outer segments may be associated with the disposition of microtubules within the narrow ciliary neck. The biochemistry of cilia and outer limbs of the photoreceptors is strikingly similar in many ways; $\mathbf{1 6}^{\mathbf{1 6}-20}$ thus cilia may form a useful model for the inaccessible photoreceptors.

It is of interest that there were some variations in the distribution of cilial abnormalities among the retinitis pigmentosa patients. In the group with $\mathrm{X}$-linked disease, there were no compound cilia but they had the highest proportion of axonemal abnormalities. Two are women in whom retinal functional defects are mild, ${ }^{21} 23$ implying that the degree of abnormality of photoreceptors and cilia is not necessarily the same. It seems possible that retinitis pigmentosa patients do not form a homogenous group and that one or other of the cilial abnormalities may be absent. This could be an important aid in efforts to subdivide cases of retinitis pigmentosa to assess the number and frequency of abnormal genes. ${ }^{21} 22$

The recognition that dynein arms may be absent in patients with bronchiectasis with or without situs inversus $^{25}$ and that this abnormality involves all cilia has highlighted the importance of establishing the appearances of dynein arms in normal cilia. Although there are two dynein arms on each microtubular doublet, in our series the largest number of dynein arms we have actually seen in a single cilium was 10 and in many there were three or less. The difficulty in visualising dynein arms is a technical matter relating mainly to fixation; and we have found that $2 \mathrm{mmol}$ magnesium sulphate added to the fixative, as suggested by Warner, ${ }^{24}$ increased the number of arms that could be seen.

The lack of orientation of cilia has been emphasised as an abnormality in the immotile cilia syndrome. $^{24}$ Afzelius $^{25}$ states that cilia in man are orientated within 5-25 degrees of each other. However, there was no quantitative evidence to support this statement. In our series, although $60 \%$ of cilia were within this range the variations in orientation were much more marked (Fig. 6). To our knowledge, this range of normal variation in the orientation of cilia has not been taken into account when assessments of abnormalities have been made. However, it is possible that the variation in the orientation of cilia is an artefact produced by differences in the rapidity of fixation of cells and cilia.

In the immotile cilia syndrome, absence of dynein arms $^{2}$ and of radial spokes ${ }^{6}$ apparently involved all cilia, but the increased incidence of abnormalities in cilia in retinitis pigmentosa ${ }^{1}$ were evident only when they were compared with normal variations described in this paper. The establishment of the variations in normal structure of cilia on a quantitative basis should make it possible to identify associations between cilial abnormalities and other diseases. Thus it is tempting to speculate that the association of cilial abnormalities and deafness (such as we have seen in our patients with retinitis pigmentosa) might also occur in patients without eye disease.

We thank Mr A E S Richards, FRCS, who performed the biopsies, Mr R S Barnett for photographic assistance, and Mrs B J Walters for typing the manuscript. We are also grateful to $\mathrm{Mr} B$ Jay, FRCS, and to Professor A Bird, FRCS, for allowing us to use their patients, and to Mrs M Jay and Mrs M Guest (of the British Retinitis Pigmentosa Society) for their assistance.

\section{References}

${ }^{1}$ Arden GB, Fox B. Increased incidence of abnormal nasal cilia in patients with retinitis pigmentosa. Nature 1979;279:534-6.

${ }^{2}$ Eliasson R, Mossberg B, Camner P, Afzelius BA. The immotile-cilia syndrome. A congenital ciliary abnormality as an etiologic factor in chronic airway infections and male sterility. $N$ Engl J Med 1977; 297:1-6.

${ }^{3}$ Charles TJ, Johnson N. Cilial abnormality in bronchiectasis. Lancet 1978;2:382. (Letter.)

${ }^{4}$ Lupin AJ, Misko GJ. Kartagener syndrome with abnormalities of cilia. J Otolaryngol 1978;7:95-102.

${ }^{5}$ Waite D, Wakefield JStJ, Steele R, Mackay J, Ross I, Wallace J. Cilia and sperm tail abnormalities in Polynesian bronchiectatics. Lancet 1978;2:132-3.

${ }^{6}$ Sturgess JM, Chao J, Wong J, Aspin N, Turner JAP. Cilia with defective radial spokes. $N$ Engl J Med 1979;300:53-6.

${ }^{7}$ Afzelius BA, Eliasson R, Johnsen Ø, Lindholmer C. Lack of dynein arms in immotile human spermatozoa. $J$ Cell Biol 1975;66:225-32.

${ }^{8}$ Pedersen $\mathrm{H}$, Rebbe $\mathrm{H}$. Absence of arms in the axoneme of immobile human spermatozoa. Biol Reprod 1975;12:541-4.

${ }^{9}$ Usher $\mathbf{C H}$. On the inheritance of retinitis pigmentosa with notes of cases. $R$ Lond Hosp Ophthalmol Rep 1914;19:130-236. 
${ }^{10}$ Fawcett DW, Porter KR. A study of the fine structure of ciliated epithelia. J Morphol 1954;94:221-81.

11 Ailsby RL, Ghadially FN. Atypical cilia in human bronchial mucosa. J Pathol 1973;109:75-8.

12 McDowell EM, Barrett LA, Harris CC, Trump BF. Abnormal cilia in human bronchial epithelium. Arch Pathol Lab Med 1976;100:429-36.

${ }^{13}$ Friedmann I, Bird ES. Ciliary structure, ciliogenesis, microvilli (Electron microscopy of the mucosa of the upper respiratory tract). Laryngoscope 1971; $81: 1852-68$.

14 Watson JHL, Brinkman GL. Electron microscopy of the epithelial cells of normal and bronchitic human bronchus. Am Rev Respir Dis 1964;90:851-66.

${ }^{15}$ De Robertis E. Electron microscope observations on the submicroscopic organization of the retinal rods. $J$ Biophys Biochem Cytol 1956;2:319-30.

${ }^{16}$ Garbers DL, Lust WD, First NL, Lardy HA. Effects of phosphodiesterase inhibitors and cyclic nucleotides on sperm respiration and motility. Biochemistry $1971 ; 10: 1825-32$.

17 Gibbons BH, Gibbons IR. Flagellar movement and adenosine triphosphatase activity in sea urchin sperm extracted with triton X-100. J Cell Biol 1972; 54:75-971

${ }^{18}$ Gray JP, Hardman JG, Bibring T, Sutherland EW. High guanyl cyclase activity in sea urchin spermatozoa. Fedn Proc 1970;29:608. (Abstract.)
${ }^{19}$ Liebman PA, Pugh EN, Jr. The control of phosphodiesterase in rod disk membranes: kinetics, possible mechanisms and significance for vision! Vision Res 1979;19:375-80.

${ }^{20}$ Stephens RE, Renaud FL, Gibbons IR. Guanine nucleotide associated with the protein of the outer fibers of flagella and cilia. Science 1967;156:1606-8.

21 Bird AC. X-linked retinitis pigmentosa. Br J Ophthalmol 1975;59:177-99.

22 Jay B, Bird A. X-linked retinitis pigmentosa. Trans Am Acad Ophthalmol Otolaryngol 1973;77:641-51.

${ }^{23}$ Berson EL, Rosen JB, Simonoff EA. Electroretinographic testing as an aid in detection of carriers of $\mathrm{X}$-chromosome-linked retinitis pigmentosa. Am J Ophthalmol 1979;87:460-8.

24 Warner FD. Cation-induced attachment of ciliary dynein cross-bridges. J Cell Biol 1978;77:R19-R26.

${ }^{25}$ Afzelius BA. The role of cilia in man. In: Perry SV, Margreth A, Adelstein RS, eds. Contractile Systems in Non-muscle Tissues. Amsterdam: Elsevier/North Holland Biomedical Press, 1976: 275-82.

Requests for reprints to: Dr B Fox, Department of Histopathology, Charing Cross Hospital Medical School, Fulham Palace Road, London W6 8RF, UK. 